\title{
Properties of Axial Diameters of a Simplex
}

\section{Mikhail Nevskii}

Received: 22 March 2010 / Revised: 14 February 2011 / Accepted: 17 April 2011 /

Published online: 7 May 2011

(C) Springer Science+Business Media, LLC 2011

\begin{abstract}
Let $S$ be a nondegenerate simplex in $\mathbb{R}^{n}$. It is proved that the minimal possible $\sigma>0$, such that a homothetic copy of $S$ of ratio $\sigma$ contains $[0,1]^{n}$, is equal to $\sum_{i=1}^{n} 1 / d_{i}(S)$. Here $d_{i}(S)$ denotes the length of a longest segment in $S$ parallel to the $i$ th coordinate axis.
\end{abstract}

Keywords $n$-dimensional simplex $\cdot n$-dimensional cube $\cdot$ Translate $\cdot$ Ratio of homothety $\cdot$ Axial diameter

\section{Introduction}

Let $n$ be a natural number and let $\mathbb{R}^{n}$ be Euclidean $n$-dimensional space. The standard form of an element $x \in \mathbb{R}^{n}$ is $x=\left(x_{1}, \ldots, x_{n}\right)$. By $\operatorname{vol}(C)$ we denote the volume of a convex body $C \subset \mathbb{R}^{n}$. By $\sigma C$ we mean the homothetic copy of $C$ of ratio $\sigma$ and homothety center at the center of gravity of $C$. The symbol $Q_{n}$ denotes the unit $n$ dimensional cube $[0,1]^{n}$. Throughout the paper $\|\cdot\|$ denotes the standard Euclidean norm in $\mathbb{R}^{n}$.

Let $S$ be a nondegenerate simplex in $\mathbb{R}^{n}$. Suppose that $x^{(j)}=\left(x_{1}^{(j)}, \ldots, x_{n}^{(j)}\right)$, $j=1, \ldots, n+1$, are the vertices of $S$. Consider the matrix

$$
\mathbf{A}=\left(\begin{array}{cccc}
x_{1}^{(1)} & \ldots & x_{n}^{(1)} & 1 \\
x_{1}^{(2)} & \ldots & x_{n}^{(2)} & 1 \\
\vdots & \vdots & \vdots & \vdots \\
x_{1}^{(n+1)} & \ldots & x_{n}^{(n+1)} & 1
\end{array}\right)
$$

M. Nevskii ( $\varangle)$

Department of Mathematics, Yaroslavl State University, Sovetskaya, 14, Yaroslavl, 150000, Russia e-mail: mnevsk@uniyar.ac.ru 
We have $\operatorname{vol}(S)=|\Delta| / n$ !, where $\Delta=\operatorname{det}(\mathbf{A})$. Since $\operatorname{vol}(S) \neq 0, \mathbf{A}$ is nondegenerate. Denote by $\Delta_{j}(x)$ the determinant that appears from $\Delta$ after replacing the $j$ th row by the row $\left(x_{1}, \ldots, x_{n}, 1\right)$. By definition, we put $\lambda_{j}(x)=\Delta_{j}(x) / \Delta$. Polynomials $\lambda_{j}$ have a property $\lambda_{j}\left(x^{(k)}\right)=\delta_{j}^{k}$, where $\delta_{j}^{k}$ is the Kronecker delta-symbol. Coefficients of $\lambda_{j}$ form the $j$ th column of $\mathbf{A}^{-1}$. Further assume that $\mathbf{A}^{-1}=\left(l_{i j}\right)$, i.e.,

$$
\lambda_{j}(x)=l_{1 j} x_{1}+\cdots+l_{n j} x_{n}+l_{n+1, j} .
$$

By the Cramer formulas,

$$
\left(\begin{array}{ccc}
x_{1}^{(1)} & \ldots & x_{1}^{(n+1)} \\
\vdots & \vdots & \vdots \\
x_{n}^{(1)} & \ldots & x_{n}^{(n+1)} \\
1 & \ldots & 1
\end{array}\right)\left(\begin{array}{c}
\lambda_{1}(x) \\
\vdots \\
\lambda_{n}(x) \\
\lambda_{n+1}(x)
\end{array}\right)=\left(\begin{array}{c}
x_{1} \\
\vdots \\
x_{n} \\
1
\end{array}\right) .
$$

Therefore, for every $x \in \mathbb{R}^{n}$,

$$
\sum_{j=1}^{n+1} \lambda_{j}(x) x^{(j)}=x, \quad \sum_{j=1}^{n+1} \lambda_{j}(x)=1 .
$$

The numbers $\lambda_{1}(x), \ldots, \lambda_{n+1}(x)$ are called the barycentric coordinates of $x$ with respect to $S$. The $(n-1)$-dimensional hyperplanes containing faces of $S$ satisfy the equations $\lambda_{j}(x)=0$. We have $S=\left\{x \in \mathbb{R}^{n}: \lambda_{j}(x) \geq 0, j=1, \ldots, n+1\right\}$.

By $d_{i}(S)$ we denote the length of a longest segment in $S$ parallel to the $i$ th axis of the coordinate system. The number $d_{i}(S)$ is called the ith axial diameter of $S$. The term axial diameter was introduced by Scott [4, 5]. In [3] the author proved that for $i=1, \ldots, n$ we have

$$
\frac{1}{d_{i}(S)}=\frac{1}{2} \sum_{j=1}^{n+1}\left|l_{i j}\right| .
$$

Moreover, $S$ contains exactly one segment of length $d_{i}(S)$ parallel to the $i$ th coordinate axis. The center of this segment coincides with the point $\sum_{j=1}^{n+1} m_{i j} x^{(j)}$, where $m_{i j}=\left|l_{i j}\right| / \sum_{k=1}^{n+1}\left|l_{i k}\right|$. Each facet of $S$ contains at least one of the endpoints of this segment. The sum of dimensions of two faces containing the endpoints of the segment is at most $n-1$.

Let $\alpha(S)$ be the minimal possible $\sigma>0$ such that a homothetic copy of $S$ of ratio $\sigma$ contains $Q_{n}$. The aim of this paper is to find out the connection between $\alpha(S)$ and the axial diameters $d_{i}(S)$. Our main result (Theorem 4 ) is the equality

$$
\alpha(S)=\sum_{i=1}^{n} \frac{1}{d_{i}(S)}
$$

We note also that $\alpha(S)$ can be found by simple computation with the coefficients $l_{i j}$ of the polynomials $\lambda_{j}$, i.e., the entries of $\mathbf{A}^{-1}$ (Corollary 3 ). 


\section{Inequality $\sum_{i=1}^{n} 1 / d_{i}(S) \leq \alpha(S)$}

First we prove the following theorem.

Theorem 1 Let $P \subset \mathbb{R}^{n}$ be a nondegenerate parallelotope and let y be a vertex of $P$. Denote the vectors determining the edges of $P$ at $y$ by $v^{(1)}, \ldots, v^{(n)}$. Let $a_{i}$ be the length of $v^{(i)}$, i.e., $a_{i}=\left\|v^{(i)}\right\|$. Let $S$ be an $n$-dimensional simplex. Denote the length of a longest segment in $S$ parallel to $v^{(i)}$ by $\delta_{i}$. If $S$ contains $P$, then

$$
\sum_{i=1}^{n} \frac{a_{i}}{\delta_{i}} \leq 1
$$

Proof Every two nondegenerate simplices in $\mathbb{R}^{n}$ are affinely equivalent. So, we can map our simplex $S$, using the affine transformation, to the simplex given by the inequalities

$$
x_{1} \geq 0, \ldots, x_{n} \geq 0, \quad \sum_{k=1}^{n} x_{k} \leq 1 .
$$

The parallelotope $P$ mapped by the same affine transformation corresponds to a parallelotope inside the special simplex defined by the inequalities above. Since affine transform preserves ratios of lengths in any direction, it is sufficient to prove (4) for mapped simplex and parallelotope. Let us denote them by the same symbols $S$ and $P$. From now on, we suppose $S$ is given by (5).

Some translate of $P$ contained in $S$ (maybe, $P$ itself) has a vertex in the hyperplane $\sum_{k=1}^{n} x_{k}=1$. Without loss of generality, we can assume that $y$, a vertex of $P$, satisfies $\sum_{k=1}^{n} y_{k}=1$.

Denote the sum of absolute values of the negative coordinates of $v^{(i)}$ by $\sigma_{i}$ and the sum of all nonnegative coordinates of $v^{(i)}$ by $\tau_{i}$, i.e.,

$$
\sigma_{i}=\sum_{k: v_{k}^{(i)}<0}\left|v_{k}^{(i)}\right|, \quad \tau_{i}=\sum_{k: v_{k}^{(i)} \geq 0} v_{k}^{(i)} .
$$

Since $\sum_{k=1}^{n} y_{k}=1$, we note that $v^{(1)}, \ldots, v^{(n)}$ belong to the closed subspace $\sum_{k=1}^{n} x_{k} \leq 0$. So, $\sum_{k=1}^{n} v_{k}^{(i)} \leq 0$ for any $v^{(i)}$ where $1 \leq i \leq n$. It means that $0 \leq \tau_{i} \leq \sigma_{i}$. The condition $v_{i} \neq 0$ implies $\sigma_{i}>0$.

By the inclusion $P \subset S$, it follows that, for each $A \subset\{1, \ldots, n\}$ and $j \in\{1, \ldots, n\}$,

$$
y_{j}+\sum_{i \in A} v_{j}^{(i)} \geq 0,
$$

holds, where $y_{j}$ and $v_{j}^{(i)}$ denote the $j$ th coordinates of $y$ and $v^{(i)}$, respectively. Hence, for each $A$,

$$
\sum_{i \in A} v_{j}^{(i)} \geq-y_{j}
$$


Now consider the sum $s=\sigma_{1}+\cdots+\sigma_{n}$. Let us show that $s \leq 1$. For a fixed $j \in\{1, \ldots, n\}$, let $A_{j}=\left\{i: v_{j}^{(i)}<0\right\}$. From the definition of $\sigma_{i}$,

$$
s=\sum_{k: v_{k}^{(1)}<0}\left|v_{k}^{(1)}\right|+\cdots+\sum_{k: v_{k}^{(n)}<0}\left|v_{k}^{(n)}\right| .
$$

Obviously, $s$ is the sum of absolute values of negative coordinates of all the vectors $v^{(i)}, 1 \leq i \leq n$. Hence, we can use another representation for $s$, namely

$$
s=\sum_{j=1}^{n} \sum_{i: v_{j}^{(i)}<0}\left|v_{j}^{(i)}\right|=\sum_{j=1}^{n} \sum_{i \in A_{j}}\left(-v_{j}^{(i)}\right) .
$$

From (6), we obtain the inequality $\sum_{i \in A_{j}}\left(-v_{j}^{(i)}\right) \leq y_{j}$. Thus,

$$
s=\sum_{j=1}^{n} \sum_{i \in A_{j}}\left(-v_{j}^{(i)}\right) \leq \sum_{j=1}^{n} y_{j}=1 .
$$

Define the points $z_{+}^{(i)}, z_{-}^{(i)}$ by the equalities

$$
\begin{aligned}
z_{+}^{(i)} & =\left(\frac{1}{\sigma_{i}} \max \left(v_{1}^{(i)}, 0\right), \ldots, \frac{1}{\sigma_{i}} \max \left(v_{n}^{(i)}, 0\right)\right), \\
z_{+}^{(i)} & =\left(-\frac{1}{\sigma_{i}} \min \left(v_{1}^{(i)}, 0\right), \ldots,-\frac{1}{\sigma_{i}} \min \left(v_{n}^{(i)}, 0\right)\right) .
\end{aligned}
$$

We claim that $z_{+}^{(i)}, z_{-}^{(i)} \in S$ for all $1 \leq i \leq n$. The $j$ th coordinate of $z_{+}^{(i)}$ is either 0 or $v_{j}^{(i)} / \sigma_{i}$ (in the case $v_{j}^{(i)}>0$ ). This value is not less than 0 since $\sigma_{i}>0$. The $j$ th coordinate of $z_{-}^{(i)}$ is either 0 or $-v_{j}^{(i)} / \sigma_{i}$ (in the case $v_{j}^{(i)}<0$ ); this value is also not less than 0 . Furthermore,

$$
\begin{aligned}
\sum_{k=1}^{n} z_{+k}^{(i)} & =\frac{1}{\sigma_{i}} \sum_{k=1}^{n} \max \left(v_{k}^{(i)}, 0\right) \\
& =\frac{1}{\sigma_{i}} \sum_{k: v_{k}^{(i)} \geq 0} v_{k}^{(i)}=\frac{\tau_{i}}{\sigma_{i}} \leq 1
\end{aligned}
$$

since $0 \leq \tau_{i} \leq \sigma_{i}$. Also we have

$$
\begin{aligned}
\sum_{k=1}^{n} z_{-k}^{(i)} & =-\frac{1}{\sigma_{i}} \sum_{k=1}^{n} \min \left(v_{k}^{(i)}, 0\right) \\
& =-\frac{1}{\sigma_{i}} \sum_{k: v_{k}^{(i)}<0} v_{k}^{(i)}=\frac{1}{\sigma_{i}} \sum_{k: v_{k}^{(i)}<0}\left|v_{k}^{(i)}\right|=\frac{\sigma_{i}}{\sigma_{i}}=1 .
\end{aligned}
$$


From above, $S=\left\{x=\left(x_{1}, \ldots, x_{n}\right): x_{k} \geq 0, \sum_{k=1}^{n} x_{k} \leq 1\right\}$. Consequently, we observe that $z_{+}^{(i)}, z_{-}^{(i)} \in S$.

Now consider the segment $\left[z_{-}^{(i)}, z_{+}(i)\right] \subset S$. Since $z_{+}^{(i)}-z_{-}^{(i)}=\left(1 / \sigma_{i}\right) v^{(i)}$, this segment is parallel to $v^{(i)}$. The length of the segment is equal to

$$
\left\|z_{+}^{(i)}-z_{-}^{(i)}\right\|=\frac{\left\|v^{(i)}\right\|}{\sigma_{i}}=\frac{a_{i}}{\sigma_{i}} .
$$

This value is not greater than $\delta_{i}$, the length of the longest line segment in $S$ parallel to $v^{(i)}$. Thus, $a_{i} / \sigma_{i} \leq \delta_{i}$, i.e., $a_{i} / \delta_{i} \leq \sigma_{i}$ for all $1 \leq i \leq n$. Therefore, we obtain

$$
\sum_{i=1}^{n} \frac{a_{i}}{\delta_{i}} \leq \sum_{i=1}^{n} \sigma_{i}=s \leq 1 .
$$

This completes the proof of the theorem.

At first the author gave another proof of Theorem 1, see [3; Corollary 4.2]. The essential idea of his first approach was the application of the equality (3) for the coefficients $l_{i j}$ of the polynomials $\lambda_{j}$. In the present paper the proof of Theorem 1 uses the ideas of the new approach that was verbally communicated to the author by V.L. Dol'nikov.

Corollary 1 Under the conditions of the above theorem, $\delta_{i} \geq n a_{i}$ for some $i=$ $1, \ldots, n$. In other words, the simplex $S$ contains a segment of length $n a_{i}$ parallel to $v^{(i)}$.

Proof By Theorem 1, $\sum_{k=1}^{n} a_{k} / \delta_{k} \leq 1$. Obviously,

$$
\frac{n a_{i}}{\delta_{i}} \leq \sum_{k=1}^{n} \frac{a_{k}}{\delta_{k}} \leq 1
$$

for some $i$. Thus $\delta_{i} \geq n a_{i}$.

Let $S$ be a nondegenerate simplex in $\mathbb{R}^{n}$. Denote by $\alpha(S)$ the minimal $\sigma>0$ satisfying $Q_{n} \subset \alpha(S) S+t$ for some $t \in \mathbb{R}^{n}$. By this definition, $\alpha(S) S$ is the smallest positive homothetic copy of $S$ that can cover the standard unit cube $Q_{n}$. Recall that $d_{i}(S)$ denotes the $i$ th axial diameter of $S$.

Corollary 2 If $S$ is a nondegenerate simplex in $\mathbb{R}^{n}$, then

$$
\sum_{i=1}^{n} \frac{1}{d_{i}(S)} \leq \alpha(S) .
$$

Proof By the definition of $\alpha(S)$, there exists $t$ such that $Q_{n} \subset \alpha(S) S+t$. Hence, for some $t^{\prime}$, we have $(1 / \alpha(S)) Q_{n}+t^{\prime} \subset S$. (If $\alpha(S)=1$, then the latter inclusion holds with $t^{\prime}=-t$.) Denote the cube $(1 / \alpha(S)) Q_{n}$ by $Q^{\prime}$. Since $Q^{\prime}$ is a parallelotope, 
we can apply Theorem 1 . In this case the vectors $v^{(i)}$ which determine edges for $Q^{\prime}$ are parallel to the coordinate axes. Thus, $\delta_{i}=d_{i}(S)$. For any vector $v^{(i)}$, holds $a_{i}=\left\|v^{(i)}\right\|=\alpha(S)^{-1}$. Inequality (4) yields

$$
\sum_{i=1}^{n} \frac{\alpha(S)^{-1}}{d_{i}(S)} \leq 1 .
$$

Multiplying the latter inequality by $\alpha(S)$, we get (7).

\section{Computation of $\alpha(S)$}

In this section we obtain the main result of the paper. Our aim is to show that the inequality in (7) can be replaced by the equality for every nondegenerate simplex $S \subset \mathbb{R}^{n}$. We prove this statement by several steps (Theorems 2-4).

Suppose $Q_{n} \not \subset S$. By definition, put $\xi(S)=\min \left\{\sigma \geq 1: Q_{n} \subset \sigma S\right\}$. It was proved in [3; Theorem 4.1] that

$$
\sum_{i=1}^{n} \frac{1}{d_{i}(S)} \leq \xi(S)
$$

and the equality in (8) is equivalent to the condition

$$
\max _{x \in \operatorname{ver}\left(Q_{n}\right)}\left(-\lambda_{1}(x)\right)=\cdots=\max _{x \in \operatorname{ver}\left(Q_{n}\right)}\left(-\lambda_{n+1}(x)\right) .
$$

Here $\operatorname{ver}\left(Q_{n}\right)$ denotes the set of vertices of $Q_{n}$. Since $\alpha(S) \leq \xi(S)$, (8) immediately follows from (7). But in the sequel (see the proof of Theorem 2 below) we essentially make use of the special condition (9) that implies the equality in (8). We start with the geometric approach to (9).

Lemma 1 Let $Q_{n} \not \subset S$ and let $1 \leq j \leq n$. Suppose the $j$ th facet of the simplex $\xi(S) S$ (parallel to the facet of $S$ satisfying the equation $\lambda_{j}(x)=0$ ) contains a vertex of $Q_{n}$. Then

$$
\xi(S)=(n+1) \max _{x \in \operatorname{ver}\left(Q_{n}\right)}\left(-\lambda_{j}(x)\right)+1
$$

Proof Since the $j$ th facet of $S$ satisfies the equation $\lambda_{j}(x)=0$ and $\xi(S)>1$, the $j$ th facet of $\xi(S) S$ satisfies the equation $-\lambda_{j}(x)=\mu$, where $\mu>0$. Denote by $w$ the vertex of $Q_{n}$ which is contained in the $j$ th facet of $\xi(S) S$. Let $c$ be the center of gravity of $S$. Let $b$ be the common point of the segment $[c, w]$ and the boundary of $S$. From the definition of $\xi(S)$, it follows that

$$
\xi(S)=\frac{\|w-c\|}{\|b-c\|} .
$$

The points $c, b, w$ lie on the common line. Since $\lambda_{j}(x)$ is the polynomial of degree 1 , it follows that

$$
\xi(S)=\frac{\lambda_{j}(w)-\lambda_{j}(c)}{\lambda_{j}(b)-\lambda_{j}(c)} .
$$


Let us prove (12). For brevity, we set $\xi=\xi(S)$. From (11),

$$
w=\frac{\|w-c\|}{\|b-c\|}(b-c)+c=\xi(b-c)+c .
$$

Since $\lambda_{j}(x)=l_{1 j} x_{1}+\cdots+l_{n j} x_{n}+l_{n+1, j}$, see (1), we have

$$
\begin{aligned}
\frac{\lambda_{j}(w)-\lambda_{j}(c)}{\lambda_{j}(b)-\lambda_{j}(c)} & =\frac{\lambda_{j}(\xi(b-c)+c)-\lambda_{j}(c)}{\lambda_{j}(b)-\lambda_{j}(c)} \\
& =\frac{\sum_{i=1}^{n} l_{i j}\left(\xi\left(b_{i}-c_{i}\right)+c_{i}\right)+l_{n+1, j}-\sum_{i=1}^{n} l_{i j} c_{i}-l_{n+1, j}}{\sum_{i=1}^{n} l_{i j}\left(b_{i}-c_{i}\right)} \\
& =\frac{\xi \sum_{i=1}^{n} l_{i j}\left(b_{i}-c_{i}\right)}{\sum_{i=1}^{n} l_{i j}\left(b_{i}-c_{i}\right)}=\xi .
\end{aligned}
$$

So, (12) is obtained. Now we need to rewrite this equality in the required form (10). Notice that $b$ is contained in the $j$ th facet of $S$, i.e., the facet satisfying the equation $\lambda_{j}(x)=0$. Hence, $\lambda_{j}(b)=0$. The center of gravity of $S$ is defined by the equality

$$
c=\frac{1}{n+1} \sum_{k=1}^{n+1} x^{(k)}
$$

where $x^{(1)}, \ldots, x^{(n+1)}$ are the vertices of $S$. By the uniqueness of the barycentric coordinates of $c$ with respect to $S$ and from (2), we have

$$
\lambda_{1}(c)=\cdots=\lambda_{n+1}(c)=\frac{1}{n+1} .
$$

So, $\lambda_{j}(c)=1 /(n+1)$. Since $Q_{n} \subset \xi(S) S$, there are no vertices of $Q_{n}$ outside $\xi(S) S$. It means that $-\lambda_{j}(w)=\mu \geq-\lambda_{j}(x)$, for all $x \in \operatorname{ver}\left(Q_{n}\right)$. We get $-\lambda_{j}(w)=$ $\max _{x \in \operatorname{ver}\left(Q_{n}\right)}\left(-\lambda_{j}(x)\right)$. Thus, it follows from (12) that

$$
\begin{aligned}
\xi(S) & =\frac{\lambda_{j}(w)-\lambda_{j}(c)}{\lambda_{j}(b)-\lambda_{j}(c)}=\frac{\lambda_{j}(w)-1 /(n+1)}{-1 /(n+1)} \\
& =(n+1) \max _{x \in \operatorname{ver}\left(Q_{n}\right)}\left(-\lambda_{j}(x)\right)+1 .
\end{aligned}
$$

The lemma is proved.

By Lemma 1, we immediately obtain the following result.

Lemma 2 Let $S$ be a nondegenerate simplex in $\mathbb{R}^{n}$. Suppose $Q_{n} \not \subset S$. If each facet of the simplex $\xi(S) S$ contains a vertex of $Q_{n}$, then the condition (9) holds.

Proof By Lemma 1, (10) holds true for each $1 \leq j \leq n+1$. We observe that $\max _{x \in \operatorname{ver}\left(Q_{n}\right)}\left(-\lambda_{j}(x)\right)$ does not depend on $j$. Therefore, $(9)$ is valid. 
Now let us show that the equality in (7) occurs in the case when any translate of $S$ does not contain $Q_{n}$.

Theorem 2 Let $S$ be a nondegenerate simplex in $\mathbb{R}^{n}$. If $Q_{n} \not \subset S+t$ for all $t \in \mathbb{R}^{n}$, then

$$
\sum_{i=1}^{n} \frac{1}{d_{i}(S)}=\alpha(S) .
$$

Proof It follows from the definition of $\alpha(S)$ that there exists $t$ such that the simplex $S_{1}=S+t$ satisfies $Q_{n} \subset \alpha(S) S_{1}$. Also the condition of the theorem implies $Q_{n} \not \subset S_{1}$. Clearly, $\alpha\left(S_{1}\right)=\alpha(S)$. From the definition of $\xi(S)$ and the inclusion $Q_{n} \subset \alpha(S) S_{1}$ we have $\xi\left(S_{1}\right) \leq \alpha(S)$. Now note that $\xi\left(S_{1}\right) \geq \alpha\left(S_{1}\right)$. Consequently, $\alpha\left(S_{1}\right)=\xi\left(S_{1}\right)=\alpha(S)$. Consider the simplex $T=\xi\left(S_{1}\right) S_{1}=\alpha\left(S_{1}\right) S_{1}$. Each facet of $T$ contains a vertex of $Q_{n}$. (Suppose that this is not so. Then we can construct a smaller than $T$ positive homothetic copy of $S_{1}$ which still contains $Q_{n}$. This contradicts the definition of $\alpha\left(S_{1}\right)$.) Since $Q_{n} \not \subset S_{1}$ and each facet of $\xi\left(S_{1}\right) S_{1}$ contains a vertex of $Q_{n}$, we can apply Lemma 2 to $S_{1}$. It follows that the condition (9) holds true for $S_{1}$. Consequently, as was noted above, we can make use of the second part of Theorem 4.1 from [3]. This approach gives an equality

$$
\sum_{i=1}^{n} \frac{1}{d_{i}\left(S_{1}\right)}=\xi\left(S_{1}\right) .
$$

Since $d_{i}\left(S_{1}\right)=d(S)$ and $\xi\left(S_{1}\right)=\alpha(S)$, we obtain (13).

Theorem 3 Let $S \subset \mathbb{R}^{n}$ be a nondegenerate simplex.

(i)

$$
\sum_{i=1}^{n} \frac{1}{d_{i}(S)} \leq 1
$$

if and only if $Q_{n}$ is contained in some translate of $S$. (ii)

$$
\sum_{i=1}^{n} \frac{1}{d_{i}(S)}=1
$$

if and only if $Q_{n} \subset S+t$ for some $t$ and each facet of $S+t$ contains a vertex of $Q_{n}$.

Proof (i) $(\Rightarrow)$ Suppose $\sum_{i=1}^{n} 1 / d_{i}(S) \leq 1$ and assume for a contradiction that no translate of $S$ contains $Q_{n}$. By Theorem 2, we have $\sum_{i=1}^{n} 1 / d_{i}(S)=\alpha(S)$. Evidently, $\alpha(S)>1$ because $S$ and all translates of $S$ are too small to contain $S$. This is a contradiction. Hence, some translate of $S$ contains $Q_{n}$.

$(\Leftarrow)$ If $Q_{n}$ is contained in some translate of $S$, then $\alpha(S) \leq 1$. Hence, Corollary 2 yields $\sum_{i=1}^{n} 1 / d_{i}(S) \leq \alpha(S) \leq 1$. 
(ii) $(\Rightarrow)$ Suppose $\sum_{i=1}^{n} 1 / d_{i}(S)=1$. By Theorem 2, it follows that some translate of $S$ contains $Q_{n}$. (Assuming that $Q_{n} \not \subset S+t$ for all $t$, we have $\alpha(S)>1$. Then (13) implies $\sum_{i=1}^{n} 1 / d_{i}(S)>1$.) Suppose for a contradiction that some facet of this translate does not contain a vertex of $Q_{n}$. Obviously, there exists $0<\sigma<1$ such that $Q_{n} \subset \sigma S+t$ for some $t$. We obtain $\alpha(S) \leq \sigma$. Applying Corollary 2 we get

$$
\sum_{i=1}^{n} \frac{1}{d_{i}(S)} \leq \alpha(S) \leq \sigma<1 .
$$

This is a contradiction. Consequently, each facet of the translate of $S$ containing $Q_{n}$ contains a vertex of $Q_{n}$.

$(\Leftarrow)$ Assume that $Q_{n}$ is contained in some translate of $S$ and that each facet of this translate contains a vertex of $Q_{n}$. Obviously, in this case $\alpha(S)=1$. By Corollary 2,

$$
\sum_{i=1}^{n} \frac{1}{d_{i}(S)} \leq 1
$$

Suppose $\sum_{i=1}^{n} 1 / d_{i}(S)<1$. Then there exists $0<\sigma<1$ such that $\sum_{i=1}^{n} 1 / d_{i}(S) \leq$ $\sigma$. It follows that

$$
\frac{1}{\sigma} \sum_{i=1}^{n} \frac{1}{d_{i}(S)}=\sum_{i=1}^{n} \frac{1}{d_{i}(\sigma S)} \leq 1 .
$$

From (i), $Q_{n} \subset \sigma S+t$ for some $t$. It means that $\alpha(S) \leq \sigma<1$. This is a contradiction (since $\alpha(S)=1$ ). Therefore, $\sum_{i=1}^{n} 1 / d_{i}(S)=1$.

Now let us formulate the main theorem of the paper.

Theorem 4 If $S$ is a nondegenerate simplex in $\mathbb{R}^{n}$, then

$$
\sum_{i=1}^{n} \frac{1}{d_{i}(S)}=\alpha(S)
$$

Proof Let $\tau=\sum_{i=1}^{n} 1 / d_{i}(S)$. Evidently,

$$
1=\frac{1}{\tau} \sum_{i=1}^{n} \frac{1}{d_{i}(S)}=\sum_{i=1}^{n} \frac{1}{d_{i}(\tau S)} .
$$

By Theorem 3, some translate of $\tau S$ contains $Q_{n}$ and each facet of $\tau S$ contains a vertex of $Q_{n}$. Thus, we have simultaneously that $\alpha(S) \leq \tau$ and that $\alpha(S) \nless \tau$. Hence, $\alpha(S)=\tau$.

Corollary 3 Suppose $S$ is an n-dimensional nondegenerate simplex. Then

$$
\alpha(S)=\frac{1}{2} \sum_{i=1}^{n} \sum_{j=1}^{n+1}\left|l_{i j}\right|,
$$


where $l_{i j}$ are the coefficients of the polynomials $\lambda_{j}$, see (1). In other words, $\alpha(S)$ is equal to half the sum of absolute values of the entries of $\mathbf{A}^{-1}$ excepting for its last row.

Proof The equality (14) follows immediately from Theorem 4 and (3). It remains to take into account that $\mathbf{A}^{-1}=\left(l_{i j}\right)$.

\section{Another Way to Prove Theorem 4}

In his report on the first version of the paper, the referee noted that he would certainly prefer self-contained proofs. In the section above we give the approach to Theorem 4 making use of some results from [3]. Now we present another proof of this theorem. However, omitting [3], we cannot obtain the equality (14).

Let $C$ be a convex body in $\mathbb{R}^{n}$, i.e., compact convex subset of $\mathbb{R}^{n}$ that has nonempty interior. Let us define the values $d_{i}(C)$ and $\alpha(C)$ by analogy with the case $C=S$. In other words, for $i \in\{1, \ldots, n\}, d_{i}(C)$ denotes the length of the longest line segment in $C$ parallel to the $i$ th coordinate axis. By $\alpha(C)$ we denote the minimal $\sigma>0$ such that a translate of $\sigma C$ contains $Q_{n}$. By the result of Scott [4; Theorem 1], it follows that if a translate of $Q_{n}$ is inscribed in $C$, then

$$
\sum_{i=1}^{n} \frac{1}{d_{i}(C)} \geq 1
$$

In this section we prove (15) in a more general case.

Theorem 5 If $C \subset \mathbb{R}^{n}$ is a convex body, then there exists $t \in \mathbb{R}^{n}$ such that $\delta Q_{n}+t \subset$ $C$, where

$$
\delta=\left(\sum_{i=1}^{n} \frac{1}{d_{i}(C)}\right)^{-1} .
$$

Proof For brevity, put $d_{i}=d_{i}(C)$. For each $i$, denote by $D_{i}$ a segment in $C$ parallel to the $i$ th axis and having the length $d_{i}$. Denote by $V$ the convex hull of $D_{1}, \ldots, D_{n}$. Let $m^{(i)}$ be the center of $D_{i}$. Let us consider the point $m=\sum_{i=1}^{n} \mu_{i} m^{(i)}$, where $\mu_{i}=\delta / d_{i}$. Since

$$
\mu_{i}>0, \quad \sum_{i=1}^{n} \mu_{i}=\delta \sum_{i=1}^{n} \frac{1}{d_{i}}=1,
$$

$m$ is a convex combination of the points of $V$. Thus, $m \in V$. Denote by $Q$ the translate of $\delta Q_{n}$ centered in $m$. Clearly, for some $t, Q=\delta Q_{n}+t$. Let us show that $Q \subset V$. An arbitrary vertex of $Q$ has the form

$$
v=m+\left( \pm \frac{\delta}{2}, \ldots, \pm \frac{\delta}{2}\right)
$$


with some arrangement of the signs + and - . Denote the standard basis of $\mathbb{R}^{n}$ by $e^{(1)}, \ldots, e^{(n)}$. We have

$$
\begin{aligned}
v & =\sum_{i=1}^{n} \frac{\delta}{d_{i}} m^{(i)}+\delta\left( \pm \frac{1}{2}, \ldots, \pm \frac{1}{2}\right)=\delta\left[\sum_{i=1}^{n} \frac{1}{d_{i}} m^{(i)}+\sum_{i=1}^{n}\left( \pm \frac{1}{2} e^{(i)}\right)\right] \\
& =\delta \sum_{i=1}^{n} \frac{1}{d_{i}}\left(m^{(i)} \pm \frac{d_{i}}{2} e^{(i)}\right)=\sum_{i=1}^{n} \mu_{i}\left(m^{(i)} \pm \frac{d_{i}}{2} e^{(i)}\right) .
\end{aligned}
$$

By the definition of $D_{i}$, we observe that $m^{(i)} \pm\left(d_{i} / 2\right) e^{(i)} \in D_{i}$. Obviously, $m^{(i)} \pm$ $\left(d_{i} / 2\right) e^{(i)}$ are the endpoints of $D_{i}$. Thus, each vertex of $Q$ is the convex combination of the endpoints of $D_{1}, \ldots, D_{n}$. Therefore, we have $Q \subset V$. Since $V=$ $\operatorname{conv}\left(D_{1}, \ldots, D_{n}\right) \subset C$, we obtain $Q \subset C$. The theorem is proved.

Corollary 4 Let $C$ be a convex body in $\mathbb{R}^{n}$ and let there exists $t^{*} \in \mathbb{R}^{n}$ such that $Q_{n}+t^{*} \subset C$. If $\sigma Q_{n}+t \not \subset C$, for all $\sigma>1$ and $t \in \mathbb{R}^{n}$, then (15) holds.

Proof Assume for a contradiction that $\sum_{i=1}^{n} 1 / d_{i}(C)<1$. Denote $\delta=$ $\left(\sum_{i=1}^{n} 1 / d_{i}(C)\right)^{-1}$. By Theorem 5, $C$ contains a translate of $\delta Q_{n}$. Since $\delta>1$, this contradicts the condition of the corollary. Thus, $\sum_{i=1}^{n} 1 / d_{i}(C) \geq 1$.

Corollary 5 If $C$ is a convex body in $\mathbb{R}^{n}$, then

$$
\sum_{i=1}^{n} \frac{1}{d_{i}(C)} \geq \alpha(C) .
$$

Proof By the definition of $\alpha(\cdot)$, the convex body $C^{\prime}:=\alpha(C) C$ satisfies Corollary 4. Hence, the inequality

$$
\sum_{i=1}^{n} \frac{1}{d_{i}\left(C^{\prime}\right)} \geq 1
$$

holds. We have $d_{i}\left(C^{\prime}\right)=\alpha(C) d_{i}(C)$. Thus, (17) is equivalent to (16). This completes the proof.

Now we are able to prove Theorem 4 by the argument different from the previous one (based on Theorem 2). Consider a nondegenerate simplex $S \subset \mathbb{R}^{n}$. By Corollary $2, \sum_{i=1}^{n} 1 / d_{i}(S) \leq \alpha(S)$. Since simplex is a special type of convex body, we can apply Corollary 5 to $S$. We obtain $\sum_{i=1}^{n} 1 / d_{i}(S) \geq \alpha(S)$. Combined together, these inequalities give

$$
\sum_{i=1}^{n} \frac{1}{d_{i}(S)}=\alpha(S),
$$

i.e., the equality of Theorem 4 . 


\section{Concluding Remarks}

Now we give some final remarks. As above, by $S$ we mean a nondegenerate simplex in $\mathbb{R}^{n}$. If $S \subset Q_{n}$, then for each $i$ holds $d_{i}(S) \leq 1$. This implies $\alpha(S)=\sum 1 / d_{i}(S) \geq$ $n$. Under the assumption $S \subset Q_{n}$, the equality $\alpha(S)=n$ is equivalent to

$$
d_{1}(S)=\cdots=d_{n}(S)=1 \text {. }
$$

For example, (18) is obvious for the simplex bounded with the hyperplanes $x_{1}=$ $0, \ldots, x_{n}=0, x_{1}+\cdots+x_{n}=1$.

Consider the condition

$$
S \subset Q_{n} \subset-n S .
$$

In his interesting paper [2], Lassak showed that (19) holds true in each of the following two cases: (1) $S$ has maximum possible volume of a simplex contained in $Q_{n}$; (2) $Q_{n}$ is a parallelotope of maximum volume in $-n S$. Moreover, it was proved that (19) implies (18). The geometrical argument used in [2] is not simple.

Let us show that (18) follows easily from (19) with the use of our results. Since $Q_{n}$ is centrally symmetric, $Q_{n}$ contains some translate of $-S$. Denote this translate by $T$. The inclusion $T \subset Q_{n}$ implies $\alpha(T) \geq n$. On the other hand, $Q_{n}$ is contained in a translate of $n T$. Hence, we get $\alpha(T) \leq n$. Consequently, $\alpha(T)=n$ and $d_{i}(S)=$ $d_{i}(T)=1$.

From the results of the present paper we can also obtain the following proposition given in [1]. Let $S$ be a nondegenerate simplex, $P$ and $P_{1}$ parallelotopes in $\mathbb{R}^{n}$. Suppose $P \subset S \subset P_{1}$ and $P_{1}$ is a homothetic copy of $P$ of ratio $\sigma>0$. Then $\sigma \geq n$. It is sufficient to consider the case $P_{1}=Q_{n}$. In this case, we have $S \subset Q_{n} \subset S_{1}$, where $S_{1}$ is a translate of $\sigma S$. These inclusions imply $n \leq \alpha(S) \leq \sigma$. Hence, $\sigma \geq n$.

Acknowledgements The author is grateful to the anonymous referee for numerous valuable remarks which allowed to improve the paper in the revised form. Also he is grateful to V. L. Dol'nikov for attention to this work and to M. Lassak for useful discussion.

\section{References}

1. Balla, M.Y.: Approximation of convex bodies by parallelotopes. International Centre for Theoretical Physics, Internal report IC/87/310, Trieste (1987)

2. Lassak, M.: Parallelotopes of maximum volume in a simplex. Discrete Comput. Geom. 21, 449-462 (1999)

3. Nevskii, M.V.: On a property of $n$-dimensional simplices. Mat. Zametki 87(4), 580-593 (2010) (in Russian). English translation: Math. Notes 87(3-4), 543-555 (2010)

4. Scott, P.R.: Lattices and convex sets in space. Q. J. Math. 36(2) 359-362 (1985)

5. Scott, P.R.: Properties of axial diameters. Bull. Aust. Math. Soc. 39, 329-333 (1989) 